\title{
Compensation for the Distorted WDM Signals through Dispersion-managed Optical Links Combined with Non-midway Optical Phase Conjugation
}

\author{
Hwang-Bin Yim ${ }^{1}$ and Seong-Real Lee ${ }^{2 *}$ \\ ${ }^{1}$ Department of ICT \& Drone Technology, Gangwon State University, 270 Yeonju- \\ ro, Jumunjin-eup, Gangneung-shi, Gangwon-do, 25425, Korea \\ ${ }^{2}$ Division of Navigational Information System, Mokpo National Maritime \\ University, Haeyangdaehang-ro 91, Mokpo-si, Jeollanam-do, 58628, Korea \\ hbinyim@gmail.com,reallee@mmu.ac.kr
}

\begin{abstract}
Dispersion-managed optical link with optical phase conjugator $(O P C)$ at the midway is well known for the compensation technique of optical signal distortion due to group velocity dispersion and fiber nonlinearities. However, in this link, the midway position of $O P C$ restricts the flexible optical link configuration. That is, the OPC position has to get out of the midway for implementation of the flexible link configuration. Therefore, the proponents of this study demonstrated the compensation effect of the non-midway OPC in dispersion-managed optical link on the distorted wavelength division multiplexed (WDM) channels. It is confirmed in this study that the non-midway OPC is somewhat effective in the compensation depending on the detailed link parameters. It is also confirmed that when the OPC was moved to transmitter or receiver of WDM system from the midway, it becomes desirable to control the net residual dispersion (NRD) in one transmission section having the lower fiber span number, and simultaneously select the start fiber span of that transmission section as the position of NRD control.
\end{abstract}

Keywords: Dispersion management, Optical phase conjugation, Non-midway OPC, Residual dispersion per span, Net residual Dispersion

\section{Introduction}

A large transmission rate has been attracting increasing interest because of the rapid spread of Web organizations [1]. According to this trend, in current fiber-optic systems, the transmission distance and channel capacity continued significantly to increase by using optical amplification and wavelength-division multiplexing (WDM). The applying erbium-doped fiber amplifiers (EDFAs) into the conventional fiber links made it possible to expand the link distance. Furthermore, WDM technology aggregates optical fiber systems that can operate at bit rates up to 40 Gbps per channel [2]. These significant achievements were also contributed by the compensation techniques of optical signal distortion due to group velocity dispersion (GVD) and fiber nonlinearities.

Dispersion management $(\mathrm{DM})$ is one of the promising compensation approaches. A DM using dispersion-compensating fibers (DCFs) has a capability to offset GVD effects of transmission fibers, such as the single-mode fibers (SMFs) by periodic alternation of the sign of the dispersion along an optical line that dramatically reduces pulse broadening [3]. If fiber nonlinearity would be neglected, it would be possible to achieve transmission of return-to-zero (RZ) pulse stream combining with the dense-WDM technique $[4,5,6]$.

Received (May 15, 2018), Review Result (July 10, 2018), Accepted (July 17, 2018)

* Corresponding Author 
This also allows for slope-matching compensation, in which the dispersion and the dispersion slope of the transmission fiber were compensated for simultaneously [7]. However, the DM technique can only compensate for optical signal distortion produced by GVD.

In contrast, optical phase conjugation can simultaneously compensate for both nonlinear impairment and GVD impairment $[8,9,10]$. An optical phase conjugator (OPC) is generally placed at the midway of the link in the conventional system. By optically conjugating the phase of signal, impairments that occurred in the first half transmission section of total transmission link (before OPC) can be cancelled by impairments that occur in the second half transmission section (after OPC)[11]. However, nonlinear impairment in optical transmission systems is not fully suppressed when using only an OPC because nonlinearity cancellation by OPC requires a perfectly symmetrical distribution of power and local dispersion with respect to OPC position [8]. Fiber attenuation makes this impossible to achieve in real applications. Furthermore, the applying of the midway OPC into WDM system is more difficult, because if perfect dispersion compensation would be accomplished for a particular channel of the WDM system, other wavelength channels may encounter different amounts of cumulative dispersion proportional to their wavelength separations from the zero-average-dispersion wavelength channel [12].

Fortunately, systems combining DM and optical phase conjugation have been proposed to address these drawbacks $[13,14,15]$. Studies have demonstrated $960 \mathrm{Gbps}$ (40 Gbps $\times$ 24 channels) WDM transmission with satisfactory system performance by applying both DM and an OPC [16, 17, 18]. Nevertheless, the dispersion-managed optical link configurations are still symmetry with respect to OPC position in systems combining DM and optical phase conjugation. However, the OPC position should be unlimited for the reconfigurable optical link of the large amplifier spacing (i.e., the long fiber span length).

To the best of our knowledge, the analysis and assessment of the effect of the nonmidway OPC in the dispersion-managed link on the compensation for the distorted WDM channels have not been reported yet. The proponents of this study demonstrated the nonmidway OPC to compensate for the distorted 24 channels x 40 Gbps WDM signals in the dispersion-managed optical link, as a function of the number of fiber spans. The rest of the paper was organized as follows. Section 2 introduces the modeling and specifications of the proposed optical transmission links and WDM systems for 24 channels x 40 Gbps. The numerical assessment method was described in Section 3. In Section 4, the simulation results and our analysis were presented. And, the conclusion was addressed in Section 5.

\section{Modeling of Dispersion-managed Link and WDM System}

\subsection{Dispersion-managed Link}

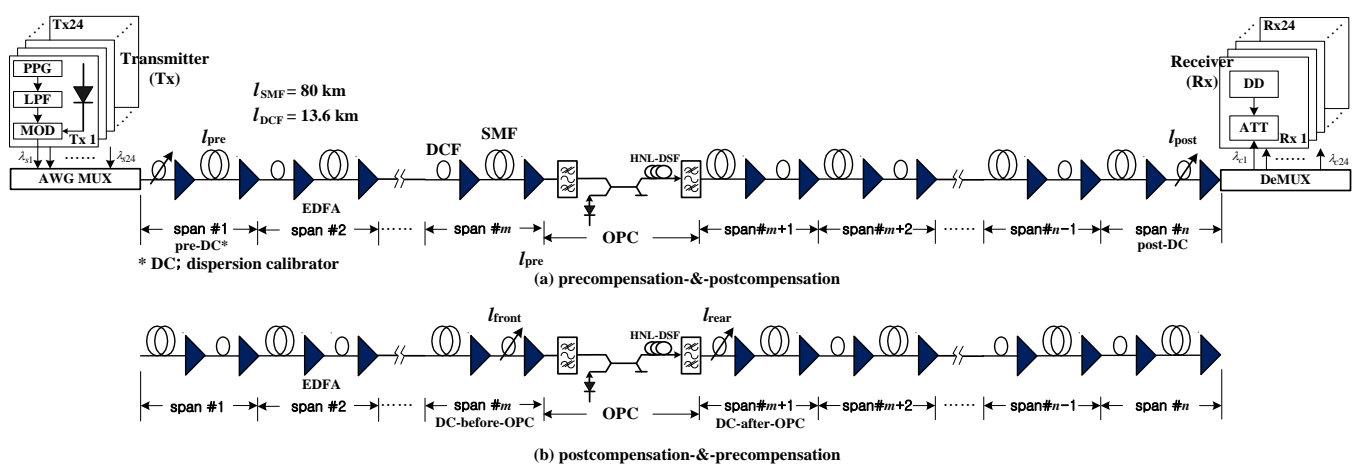

Figure 1. Configuration of Dispersion-managed Link with OPC for WDM Transmission 
The DM optical transmission link comprised $n$ fiber spans, with an OPC. The setup is shown in Figure 1. In Figure 1, if the condition of $n=2 m$ would be established, the OPC becomes the midway OPC. Total number of fiber spans, i.e., $n$, was assumed to be 10 to 200 with 10 span interval, for evaluating the effect of the number of fiber spans on the compensation of the distorted WDM signals. Also, various OPC positions were considered, including the midway represented as 'the first transmission section (FTS) versus the second transmission section (STS)', such as $3: 7,4: 6,5: 5,6: 4$, and $7: 3$ schemes.

In this paper, the compensation effect depending upon the deployment of the SMFs and the DCFs in a fiber span on the distorted WDM signals was also assessed, because optical system performance depends on the position of the DCF [19], and on the amount of residual dispersion $[20,21,22]$. From the viewpoint of DCF's position, the dispersion compensation scheme is specified into the pre- and postcompensation where the DCF is located before and after the SMF in every fiber spans, respectively. In precompensation, the DCF precedes the SMF; in postcompensation, they are reversed. As shown in Figure 1(a), precompensation and postcompensation were configured in all fiber spans before and after the OPC to allow pre-dispersion calibration (pre-DC) and post-dispersion calibration (post-DC) for use in controlling the net residual dispersion (NRD). In contrast, in Figure 1(b), postcompensation and precompensation were configured in all fiber spans to allow dispersion calibration before OPC (DC-before-OPC) and dispersion calibration after OPC (DC-after-OPC).

The length of the SMF in every configuration, $l_{\mathrm{SMF}}$, was assumed to be $80 \mathrm{~km}$. Furthermore, the SMF was characterized by attenuation coefficient $\alpha_{\mathrm{SMF}}=0.2 \mathrm{~dB} / \mathrm{km}$, dispersion coefficient $D_{\mathrm{SMF}}=17 \mathrm{ps} / \mathrm{nm} / \mathrm{km}$, and nonlinear coefficient $\gamma_{\mathrm{SMF}}=1.35 \mathrm{~W}^{-1} \mathrm{~km}^{-1}$ at $1,550 \mathrm{~nm}$. On the other hand, the DCF of every fiber span was characterized by dispersion coefficient $D_{\mathrm{DCF}}=-100 \mathrm{ps} / \mathrm{nm} / \mathrm{km}$, attenuation coefficient $\alpha_{\mathrm{DCF}}=0.6 \mathrm{~dB} / \mathrm{km}$, and nonlinear coefficient $\gamma_{\mathrm{DCF}}=5.06 \mathrm{~W}^{-1} \mathrm{~km}^{-1}$ at $1,550 \mathrm{~nm}$.

In the case of DM applied to every fiber span (i.e., the in-line DM), residual dispersion per span (RDPS) was defined as the dispersion accumulated in each fiber span, while the NRD was defined as the total dispersion accumulated at the end of the transmission link. The RDPSs of every fiber spans were assumed to be $0 \mathrm{ps} / \mathrm{nm}$, i.e., the complete in-line dispersion compensation. Thus, the length of the DCF of all fiber spans, $l_{\mathrm{DCF}}$, becomes $13.6 \mathrm{~km}$, except special fiber span for all the configurations. Though, the NRD was also 0 $\mathrm{ps} / \mathrm{nm}$ in the case of RDPS $=0 \mathrm{ps} / \mathrm{nm}$, the optimal NRD had been reported may not be 0 $\mathrm{ps} / \mathrm{nm}$ in a "pseudolinear" system [23]. Thus, it is required to induce the optimal NRD of each link configurations and to apply the induced optimal NRD into each links. The DCF of the first fiber span or the last fiber span in Figure 1(a) plays a role as the calibrator of each transmission section's NRD. Also, the DCF of the fiber span before or after OPC in Figure 1 (b) plays a role as the calibrator of each transmission section's NRD. That is, $l_{\text {pre }}$ and $l_{\text {post }}$ in Figure 1(a), and $l_{\text {front }}$ and $l_{\text {rear }}$ in Figure 1(b) were used to determine the NRD of FTS and STS, respectively.

\subsection{WDM System and OPC}

Modeling of the transmitters (Tx) in Figure 1 was based on the distributed feedback laser diodes (DFB-LD). The center wavelengths of each DFB-LD range from 1,550 nm to $1,568.4 \mathrm{~nm}$, and wavelength space between each channel was assumed to be $100 \mathrm{GHz}$ $(0.8 \mathrm{~nm})$, based on recommendation G.694.1 from the ITU-T [24]. An independent 40 Gbit/s $127\left(=2^{7}-1\right)$ pseudo random bit sequence (PRBS) externally modulate the DFBLDs, and consequently 127 return-to-zero (RZ) signals per a channel were generated through the external optical modulator. RZ pulses were assumed to be a chirp-free second-order super-Gaussian pulse with a $10 \mathrm{~dB}$ extinction ratio (ER), and a $50 \%$ duty cycle. 


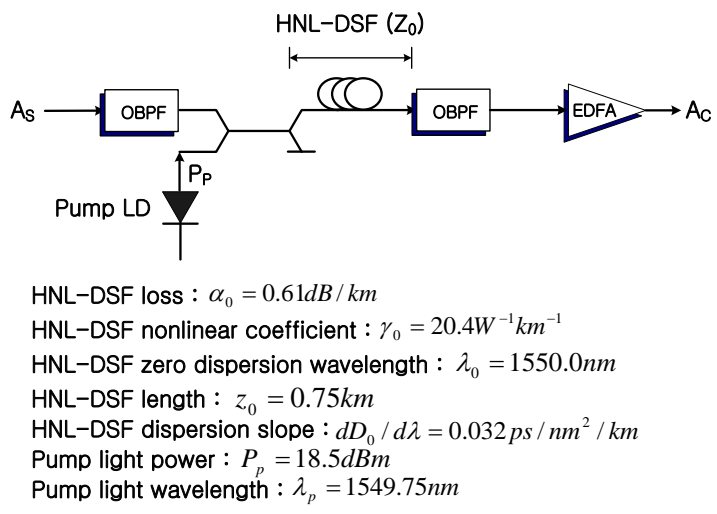

Figure 2. The Configuration and Parameters of HNL-DSF optical Phase Conjugator

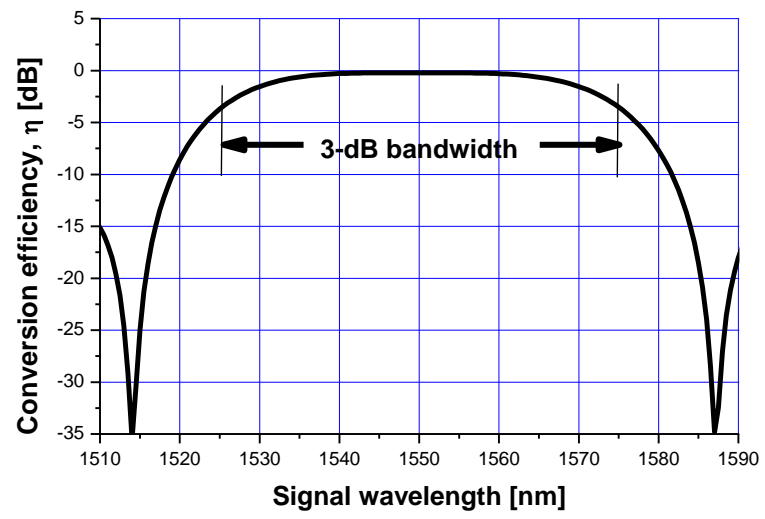

Figure 3. Conversion Efficiency of HNL-DSF Optical Phase Conjugator

Each channel was multiplexed in the arrayed waveguide grating multiplexer (AWG MUX), and then transmitted to dispersion-managed link. A highly nonlinear dispersionshifted fiber (HNL-DSF) OPC was used. The configuration of OPC and the parameters of the HNL-DSF were plotted and summarized in Figure 2. Because the wavelength of the pump light was assumed to be $1,549.75 \mathrm{~nm}$, the conjugated wavelengths of 24 channels waves range from $1549.5 \mathrm{~nm}$ to $1528.5 \mathrm{~nm}$ for $1,550-1,568.4 \mathrm{~nm}$ of the signal at the midway OPC.

The conversion efficiency $\eta$ of OPC was defined as a ratio of the four-wave mixing (FWM) product power to the input probe (signal) power [25]. The 3-dB bandwidth of $\eta$ was obtained to $48 \mathrm{~nm}(1526-1574 \mathrm{~nm})$ as shown in Figure 3. The conjugated wavelengths as well as the signal wavelengths were included in the 3-dB bandwidth.

The 24 conjugated multiplexed channels were propagated through the second half of link, demultiplexed, and sent into each receiver (Rx), for direct detection. Each Rx in Fig. 1 consists of EDFA as a preamplification stage, an optical filter, a photodetector, a pulse shaping Butterworth filter, and a decision circuit. The noise figure of EDFA was $5 \mathrm{~dB}$, and bandwidth of optical filter was $1 \mathrm{~nm}$ in this study. Modeling of the photodetector was based on the PIN diode. The receiver bandwidth is assumed to be $0.65 \times 40 \mathrm{Gbps}$ [4].

\section{Numerical Assessment}

The propagation of the signal in a lossy, dispersive, and nonlinear medium can be expressed by the nonlinear Schrödinger equation (NLSE), assuming a slowly varying envelope approximation [26]: 


$$
\frac{\partial A_{j}}{\partial z}=-\frac{\alpha}{2} A_{j}-\frac{i}{2} \beta_{2 j} \frac{\partial^{2} A_{j}}{\partial T^{2}}+\frac{1}{6} \beta_{3 j} \frac{\partial^{3} A_{j}}{\partial T^{3}}+i \gamma_{j}\left|A_{j}\right|^{2} A_{j}+2 i \gamma_{j}\left|A_{k}\right|^{2} A_{j}
$$

where $j, k=1,2, \ldots, 24(j \neq k), A_{j}$ represents the complex amplitude of the signal of the $j$-th channel, $z$ is the propagation distance, $\beta_{2 j}$ is the GVD, $\beta_{3 j}$ is third-order dispersion, $\gamma_{j}$ is the nonlinear coefficient, and $T=t-z / v_{j}$ is the time measured in a retarded frame. The last two terms of (1) induce SPM and cross-phase modulation (XPM), respectively. The effects of XPM on WDM signals decrease as the fiber dispersion increases [27]. Thus, XPM's effect on SMF links was generally neglected in the analysis of NLSE. The numerical approach of (1) was completed by using the split-step Fourier method [26].

Eye opening penalty (EOP) was used to assess the system performance of the receiving WDM signals in this work, as shown in the following equation:

$$
E O P[d B]=10 \log _{10} \frac{E O_{r e c}}{E O_{b t b}}
$$

where $E O_{\text {rec }}$ and $E O_{\text {btb }}$ were the eye opening (EO) of the receiving optical pulse and EO of the input optical pulse, respectively. EO was defined as $2 P_{\text {av }} /\left(\mathrm{P}_{1, \min }-\mathrm{P}_{0, \max }\right)$, where $P_{\mathrm{av}}$ was the averaged power of the optical signals, and $P_{1, \min }$ and $P_{0, \max }$ were the minimum power of the ' 1 ' optical pulse and the maximum power of the ' 0 ' optical pulse, respectively.

\section{Results and Discussion}
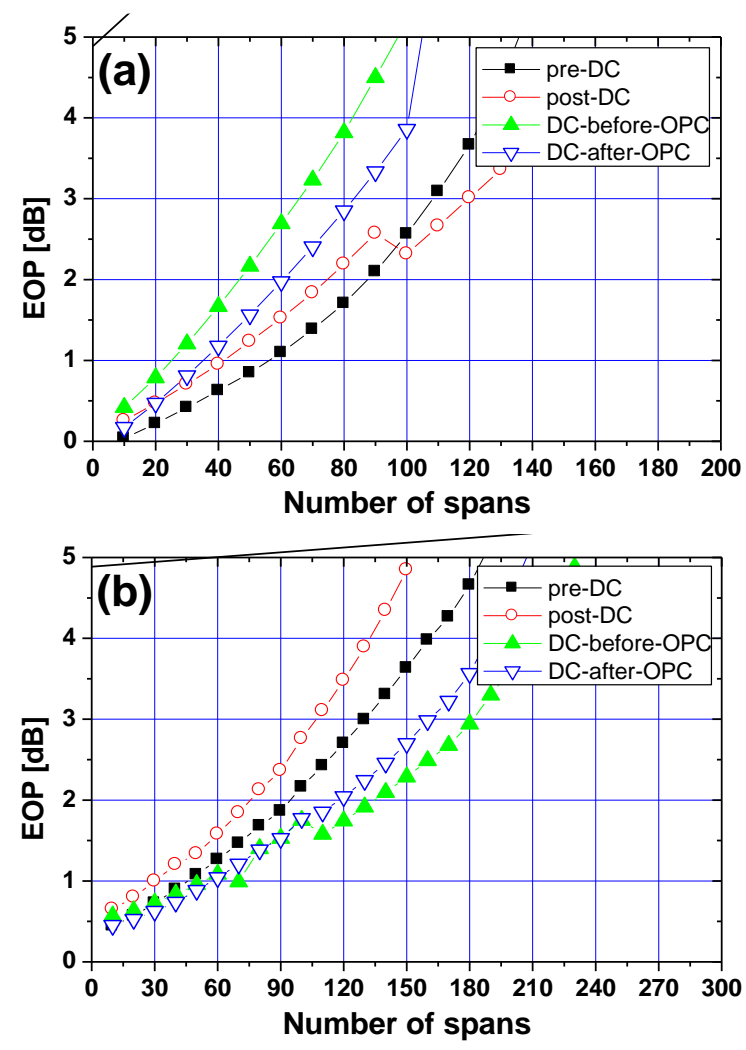

Figure 4. The EOPs of the Worst Channel as a Function of the Number of Fiber Spans in the Cases of (a) $0 \mathrm{dBm}$ Launching into the 4:6 scheme DM Link and (b) of $-5 \mathrm{dBm}$ Launching into the 7:3 scheme DM Link

It had been confirmed that the optimal NRD of the overall configuration, at which the eye opening penalty (EOP) was smallest, was found to be $10 \mathrm{ps} / \mathrm{nm}$. This is 
remarkably close to the result reported in [23] dealing with "pseudolinear" system and with author's other results reported in $[16,17,18]$. Figure 4 shows the EOPs of the worst channel with a launch power of $0 \mathrm{dBm}$ in (Figure 4(a)) and $-5 \mathrm{dBm}$ (Figure 4(b)) in DM link of 4:6 scheme and 7:3 scheme, respectively, as a function of the number of fiber spans. It can be easily demonstrated that the compensation characteristics depend on the NRD control method, the launching power, the OPC position as well as the number of fiber spans.
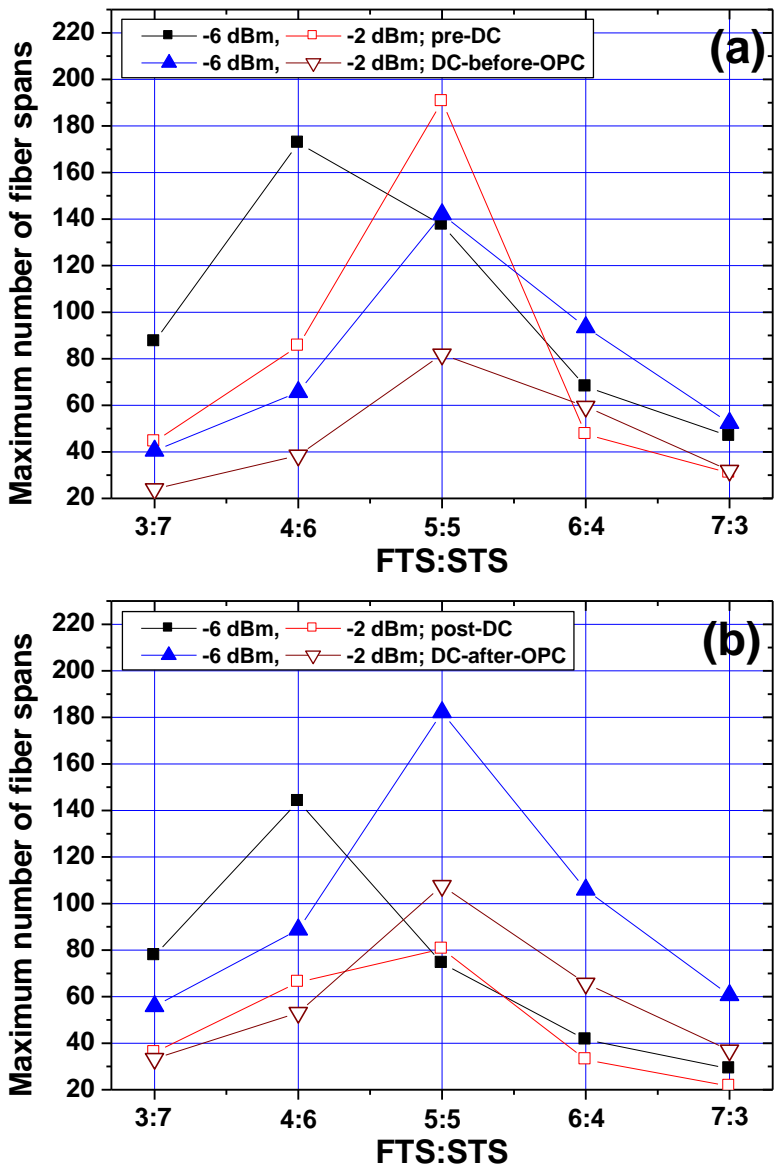

Figure 5. The Maximum Number of Fiber Span for Dispersion Calibration Methods as a Function of OPC Position Rate: (a) pre-DC and DC-before-OPC and (b) post-DC and DC-after-OPC

In fiber communication systems, $1 \mathrm{~dB}$ EOP was used as the standard system performance criterion. This is equivalent to pulse broadening (the ratio of the received pulse root-mean-square (RMS) width to the initial pulse RMS width) of 1.25 and corresponds to a $10^{-12}$ bit error rate (BER) [26]. Figure 5(a) shows the maximum number of fiber span results in the EOP of $1 \mathrm{~dB}$ in the optical link, in which the NRD controlled by the dispersion calibration at FTS (i.e., pre-DC or DCbefore-OPC), as a function of the OPC position. In contrast, Figure 5(b) shows the maximum number of fiber span results in the EOP of $1 \mathrm{~dB}$ in the optical link, in which the NRD controlled by the dispersion calibration at STS. It was easily expected that the maximum number of fiber spans was obtained in case of the midway OPC (5:5 scheme). However, in case of transmitting WDM channels of -6 $\mathrm{dBm}$ through the optical link with the NRD of $10 \mathrm{ps} / \mathrm{nm}$ by pre-DC and post-DC, the maximum number of fiber spans was obtained at 4:6 scheme rather than 5:5 scheme. 
The results shown in Figure 5 suggest that the optimal OPC position should be selected by the method of NRD control and the launch power of WDM channels.

Figure 6 (a)-(d) illustrates the effective range of launch power as a function of the number of fiber spans in the optical link with the NRD of $10 \mathrm{ps} / \mathrm{nm}$ by pre-DC (Figure 6(a), post-DC (Figure 6(b), DC-before-OPC (Figure 6(c), and DC-after-OPC (Figure 6(d), respectively. First, the proponents of this study confirm that the effective range of launch power in 4:6 scheme is wider than 5:5 scheme in case of post-DC. The noticeable result of Figure 6 is that the effective range of launch power in the optical link with OPC closer to Tx (i.e., 4:6 and 3:7) is broader than the optical link with OPC closer to Rx (i.e., 6:4 and 7:3) in cases of the NRD controlling by pre-DC and post DC. In contrast, in cases of the NRD controlling by DC-before-OPC and DC-after-OPC, the effective range of launch power in the optical link with OPC closer to Rx is broader than the optical link with OPC closer to Tx.
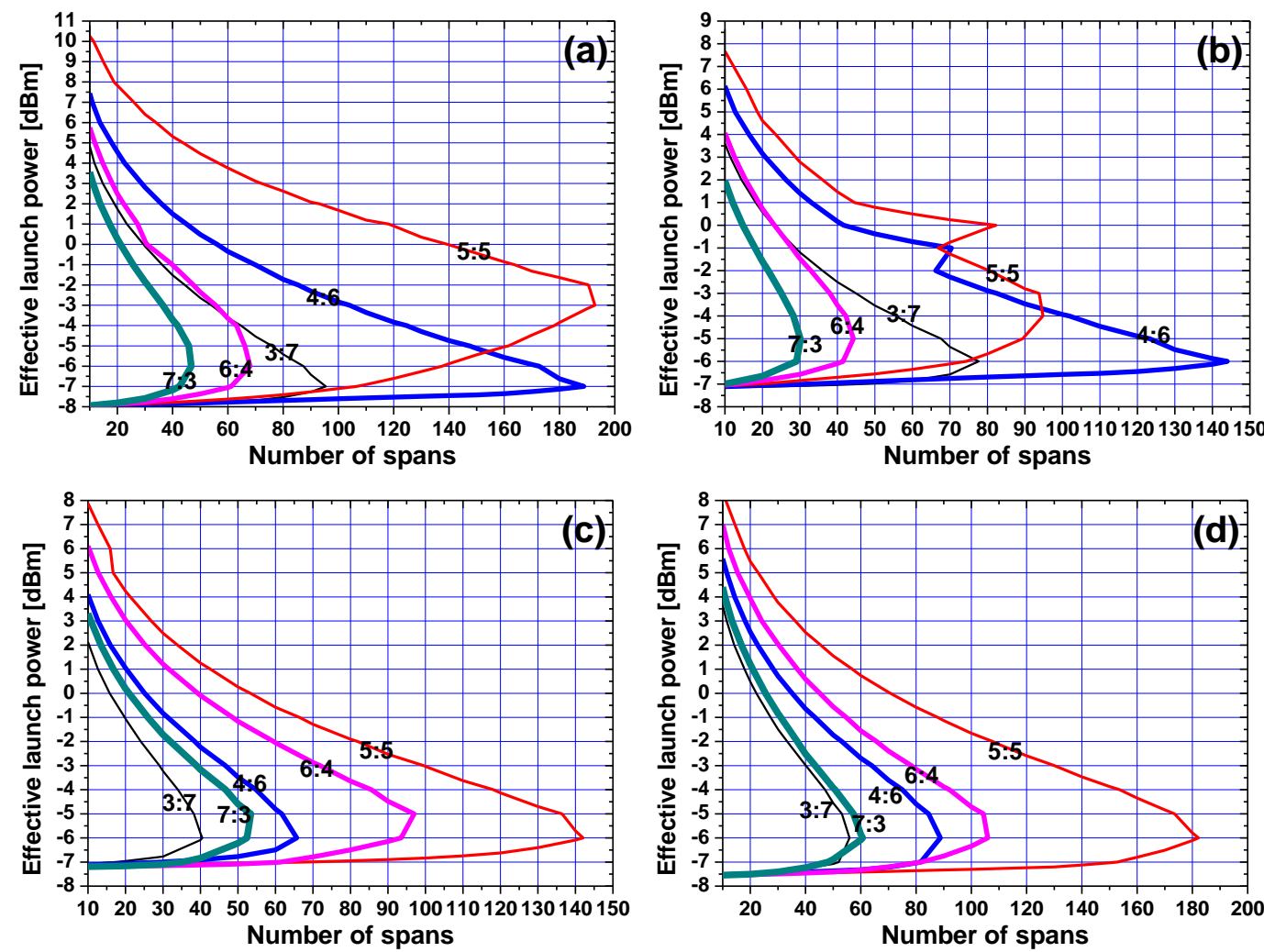

\section{Figure 6. The Effective Range of Launch Power as a Function of the Number of Fiber Span: (a) pre-DC, (b) post-DC, (c) DC-before-OPC and (d) DC-after- OPC}

It should be required to quantitatively analyze factor for the ease of comparison of the effective range of launch power affected by the NRD control methods and OPC positions. The area of contour from the results of Figure 6 was used as a quantitative factor. The area of contour was defined as 'product of span numbers and (launch) power'.

The area of contour, i.e., the product of span numbers and (launch) power was plotted in Figure 7 as a function of the OPC position and the NRD control methods. The optimal NRD control method was obtained to be pre-DC in the optical link with OPC closer to Tx, including 5:5 scheme, in contrast, the optimal NRD control method was obtained to be DC-after-OPC in the optical link with OPC closer to Rx, 
excluding 5:5 scheme. Both of pre-DC and DC-after-OPC have the position of the NRD control in each half transmission section in common. That is, in pre-DC and DC-after-OPC, the NRD was controlled at the first fiber span of FTS and STS, respectively. Consequently, when the OPC was moved to $\mathrm{Tx}$ or $\mathrm{Rx}$ from the midway, it is desirable to control the NRD in one transmission section having the lower fiber span number, and simultaneously, to select the start fiber span of that transmission section as the position of NRD control, for implementing of the flexible DM link unaffected by the OPC position.

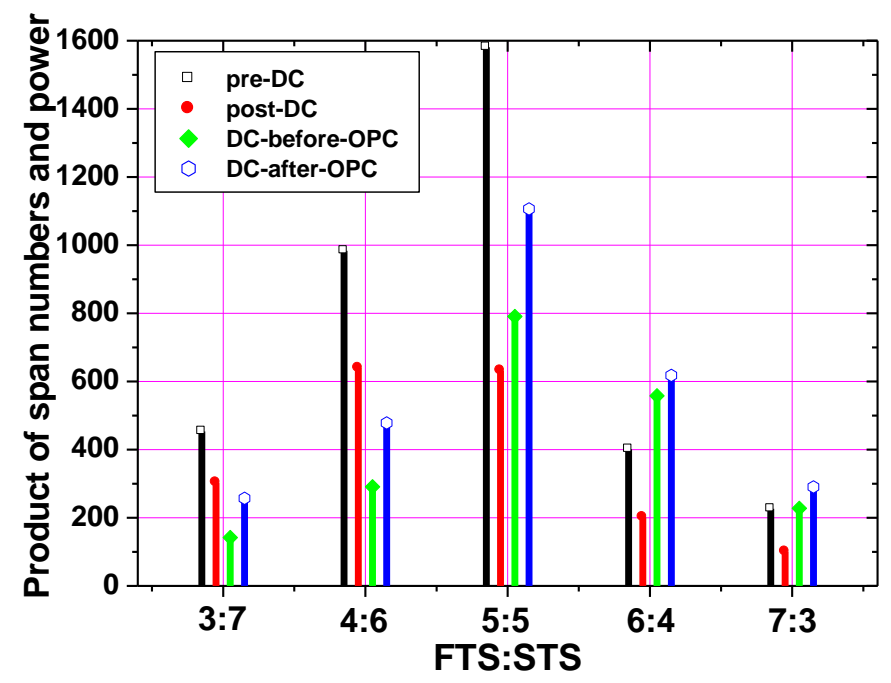

Figure 7. The Product of Span Numbers and Launch Power as a Function of the OPC Position

\section{Conclusion}

The simulations described in this paper were aimed at investigating the compensation effect of the non-midway OPC in dispersion-managed optical link on the distorted WDM channels due to GVD and fiber nonlinearities. The adopting of the non-midway OPC is for the implementation of the flexible optical links configuration. The proponents of this study confirm that though the compensation by using the non-midway OPC for the distorted WDM channels is less effective than using the midway OPC, the non-midway OPC is somewhat effective in the compensation depending on the deployment of SMF and DCF and the NRD control method. Also confirmed is that when the OPC was moved to Tx or Rx from the midway for implementing of the flexible DM link, it becomes desirable to control the NRD in one transmission section having the lower fiber span number, and simultaneously select the start fiber span of that transmission section as the position of NRD control.

\section{References}

[1] I. Nadeem and D. Y. Choi, "Concentric Core Fiber Design for Optical Fiber Communication,” Journal of Information and Communication Convergence Engineering (JICCE), vol. 14, no. 3, (2016), pp.163170.

[2] R. Cigliutti, A. Galtarossa, M. Giltrelli, D. Grosso, A. W. R. Leitch, L. Palmieri, S. Santoni, L. Schenato, D. Waswa, "Design, Estimation and Experimental Validation of Optical Polarization Mode Dispersion Compensator in 40 Gbit/s NRZ and RZ Optical Systems," Optical Fiber Technology, vol. 15, no. 3, (2009), pp. 242-250.

[3] P. M. Lushnikov, "Oscillating Tails of a Dispersion-managed Soliton," Journal of Optical Society of America B, vol. 21, no. 11, (2004), pp. 1913-1917.

[4] G. P. Agrawal, "Fiber-Optics Communication Systems", Wiley, New York, (1997). 
[5] E. Iannone, F. Matera, A. Mecozzi, and M. Settembre, "Nonlinear Optical Communication Networks", Wiley, New York, (1998).

[6] P. Y. P. Chen, B. A. Malomed, and P. L. Chu, "Stabilization of Solitons Against Timing Jitter and Collisions by Notch Filters in Multichannel Fiber-optic Links," Journal of Optical Society of America B, vol. 21, no. 4, (2004), pp. 719-728.

[7] H. Wei and D. V. Plant, "Simultaneous Nonlinearity Suppression and Wide-band Dispersion Compensation Using Optical Phase Conjugation," Optics Express, vol. 12, no. 9, (2004), pp. 1938-1958.

[8] S. Watanabe and M. Shirasaki, "Exact Compensation for Both Chromatic Dispersion and Kerr Effect in a Transmission Fiber Using Optical Phase Conjugation,” Journal of Lightwave Technology, vol. 14, no. 3, (1996), pp. 243-248.

[9] S. L. Jansen, D. van den Borne, P. M. Krummrich, S. Spälter, G.-D. Khoe, H. de Waardt, "Long-haul DWDM Transmission Systems Employing Optical Phase Conjugation," IEEE Journal of Selected Topics in Quantum, vol. 12, no. 4, (2006), pp. 505-520.

[10] X. Tang and Z. Wu, "Reduction of Intrachannel Nonlinearity Using Optical Phase Conjugation," IEEE Photonics Technology Letters, vol. 17, no. 9, (2005), pp. 1863-1865.

[11] A. Yariv, D. Fekete, and D. M. Pepper, "Compensation for Channel Dispersion by Nonlinear Optical Phase Conjugation," Optical Letters, vol. 4, (1979), pp. 52-54.

[12] A. H. Gnauck, J. M. Wiesenfeld, L. D. Garrett, M. Eiselt, F. Forghieri, L. Arcangeli, B. Agogliata, V. Gusmeroli, and D. Scarano, "16x20 Gb/s, $400 \mathrm{~km}$ WDM Transmission over NZDSF Using a Slope Compensating Fiber-grating Module," IEEE Photonics Technology Letters, vol. 12, no. 4, (2000), pp. 437-439.

[13] X. Xiao, C. Yang, S. Gao, and Y. Tian, "Partial Compensation of Kerr Nonlinearities by Optical Phase Conjugation in Optical Fiber Transmission Systems without Power Symmetry," Optics Communications. vol. 265, no. 1, (2006), pp. 326-330.

[14] A. Chowdhury and R. J. Essiambre, "Optical Phase Conjugation and Pseudolinear Transmission", Optics Letters, vol. 29, no. 10, (2004), pp. 1105-1107.

[15] P. Minzioni and A. Schiffini, "Unifying Theory of Compensation Techniques for Intrachannel Nonlinear Effects," Optics Express, vol. 13, no. 21, (2005), pp. 8460-8468.

[16] S. R. Lee, "Pseudo-Symmetrically Dispersion-managed Optical Transmission Links with the Midway OPC for compensation for the Distorted WDM Signals," Journal of Information and Communication Convergence Engineering (JICCE), vol. 13, no. 4, (2015), pp. 228-234.

[17] J. P. Chung and S. R. Lee, "The Compensation Effects of the Position of Dispersion Compensating Fiber and the Control Position of Net Residual Dispersion on the WDM Signal Distortions in the Dispersion-managed Optical Links with Optical Phase Conjugator,” Information, vol. 19, no. 1, (2016), pp. 149-156.

[18] H. B. Yim and S. R. Lee, "Compensation Characteristics of Optical Signal Distortions in Dispersionmanaged Optical Links with Randomly Distributed RDPS," International Journal of Information and Communication Technology, vol. 8, no. 1, (2016), pp. 37-49.

[19] D. M. Rothnie and J. E. Midwinter, "Improved Standard Fiber Performance by Positioning the Dispersion Compensating Fiber," Electronic Letters, vol. 32, no. 20, (1996), pp. 1907-1908.

[20] G. Bellotti, A. Bertaina, and S. Bigo, "Dependence of Self-phase Modulation Impairments on Residual Dispersion in 10 Gbit/s-based Terrestrial Transmissions Using Standard Fiber," IEEE Photonics Technology Letters, vol. 11, no. 7, (1999), pp. 824-826.

[21] R. J. Nuyts, Y. K. Park, and P. Gallion, "Performance Improvement of $10 \mathrm{~Gb} / \mathrm{s}$ Standard Fiber Transmission Systems by Using the SPM Effect in the Dispersion Compensating Fiber," IEEE Photonics Technology Letters, vol. 8, no. 10, (1996), pp. 1406-1408.

[22] C. Peucheret, N. Hanik, R. Freund, L. Molle, and P. Jeppesen, "Optimization of Pre- and PostDispersion Compensation Schemes for 10-Gbits/s NRZ Links Using Standard and Dispersion Compensating Fibers," IEEE Photonics Technology Letters, vol. 12, no. 8, (2000), pp. 992-994.

[23] R. I. Killey, H. J. Thiele, V. Mikhailov, and P. Bayvel, "Reduction of Intrachannel Nonlinear Distortion in 40-Gb/s-based WDM Transmission over Standard Fiber," IEEE Photonics Technology Letters, vol. 12, no. 12, (2000), pp. 1624-1626.

[24] ITU Recommendation "Characteristics of a non-zero dispersion shifted single-mode optical fibre cable" G.655, 2003.

[25] K. Inoue, "Four-wave mixing in an optical fiber in the zero-dispersion wavelength region," Journal of Lightwave Technology, vol. 10, no. 11, (1992), pp. 1553-1561.

[26] G. P. Agrawal, "Nonlinear Fiber Optics," Academic Press, San Francisco (2001).

[27] P. Minzioni, F. Alberti, and A. Schiffini, "Optimized Link Design for Nonlinearity Cancellation by Optical Phase Conjugation," IEEE Photonics Technology Letters, vol. 16, no. 3, (2004), pp. 813-815.

[28] N. Kikuchi and S. Sasaki, "Analytical Evaluation Technique of Self-phase Modulation Effect on the Performance of Cascaded Optical Amplifier Systems," Journal of Lightwave Technology, vol. 13, no. 5, (1995), pp. 868-878. 


\section{Authors}

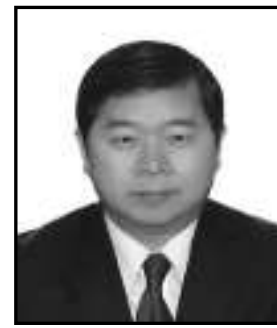

Hwang-Bin Yim received Ph.D. degrees from Soonchunhyang University, Seoul, Korea, in Information Communication Engineering in 2003. He has been working as a Professor in Gangwon State University from March 2003. His research interests include Communication Application System, Optical Communication, Electronic circuit design and so on.

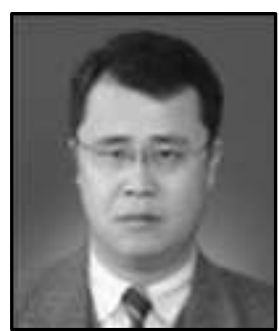

Seong-Real Lee received the B.S., M.S., and Ph.D. degrees in telecommunication and information engineering from Korea Aerospace University, Korea in 1990, 1992, and 2002, respectively. $\mathrm{He}$ is currently a professor of Division of Navigation Information System at Mokpo National Maritime University. His research interests include optical WDM systems, optical soliton systems, and optical nonlinear effects. 